\title{
Life performance of oil and gas platforms for various production profiles and feed compositions
}

Nguyen, Tuong-Van; de Oliveira Júnior, Silvio

\author{
Published in: \\ Energy
}

Link to article, DOI:

10.1016/j.energy.2018.07.121

Publication date:

2018

Document Version

Peer reviewed version

Link back to DTU Orbit

Citation (APA):

Nguyen, T-V., \& de Oliveira Júnior, S. (2018). Life performance of oil and gas platforms for various production profiles and feed compositions. Energy, 161, 583-594. https://doi.org/10.1016/j.energy.2018.07.121

\section{General rights}

Copyright and moral rights for the publications made accessible in the public portal are retained by the authors and/or other copyright owners and it is a condition of accessing publications that users recognise and abide by the legal requirements associated with these rights.

- Users may download and print one copy of any publication from the public portal for the purpose of private study or research.

- You may not further distribute the material or use it for any profit-making activity or commercial gain

- You may freely distribute the URL identifying the publication in the public portal 


\title{
Life performance of oil and gas platforms for various production profiles and feed compositions
}

\author{
Tuong-Van Nguyen ${ }^{\mathrm{a}, \mathrm{b}, *}$, Silvio de Oliveira Júnior ${ }^{\mathrm{a}}$ \\ ${ }^{a}$ Laboratory of Environmental and Thermal Engineering, Polytechnic School - University of São Paulo, Av. Prof. Luciano \\ Gualberto, 05508-900 São Paulo, Brazil \\ ${ }^{b}$ Section of Thermal Energy, Department of Mechanical Engineering, Technical University of Denmark, \\ Building 403, Nils Koppels Allé, 2800 Kongens Lyngby, Denmark
}

\begin{abstract}
Oil and gas platforms present similar structural designs but process fluids with different thermo-physical and chemical properties, and with varying flowrates (variability of the gas-to-oil and water-to-oil ratios over time). It is therefore not possible to suggest a standard flow diagram of these facilities. Different processes and operating modes may be implemented to maximize the petroleum production and improve the overall system performance. The present work evaluates, in a first step, the variations of the heating, cooling and power demands over time, in terms of energy and exergy. The simulations were calibrated using actual field data (feed compositions and production profiles). In a second step, the minimum energy and exergy losses of the platform are assessed by performing a thermodynamic analysis, assuming an ideal scenario in which all processes are run at their design points. This approach proves to be useful for evaluating consistently different options for oil and gas production, and for determining, in a further step, the most promising solutions for minimizing the energy use over a field lifetime. The compression (natural gas and carbon dioxide) processes represent the major share of the total power demand ( $\geq 80 \%)$ for all feed compositions, at all stages of the field life. The power and heat generation system is responsible for about 60 to $70 \%$ of the total exergy destruction over time, followed by the gas treatment and membrane units. Efforts should therefore focus on a more efficient design and operation of the gas compression units, which are designed to handle the peak production of hydrocarbons, and on the valorisation of the turbine exhausts. Alternative $\mathrm{CO}_{2}$-treatment processes may also be of interest for feeds with high $\mathrm{CO}_{2}$-composition.
\end{abstract}

Keywords: Oil and gas platform, energy analysis, process modelling, exergy analysis, lifetime performance

\section{Introduction}

Offshore platforms include similar process operations worldwide: oil, gas and water separation; oil stabilization; gas compression and purification; produced water treatment. Hydrocarbon processing may be

*Principal corresponding author. Tel.: +45 4525 4129

Email address: tungu@mek.dtu.dk (Tuong-Van Nguyen) 


\begin{tabular}{|llll|}
\hline Nomenclature & & \\
$T$ & Temperature, $\mathrm{K}$ or ${ }^{\circ} \mathrm{C}$ & ph & physical \\
$\dot{E}$ & Exergy rate, W & Subscripts \\
$\dot{Q}$ & Heat rate, W & 0 & dead state \\
$\dot{W}$ & Power, W & cw & cooling water \\
$p$ & Pressure, Pa & D & destruction \\
Abbreviations & eg & exhaust gases \\
EOS & Equation of State & F & fuel \\
PP & Processing Plant & feed & feed \\
PR & Peng-Robinson & iw & injected water \\
UT & Utility Plant & L & loss \\
VRU & Vapour Recovery Unit & P & product \\
Superscripts & rw & rejected water \\
ch & chemical & tot & total \\
& & & \\
\hline
\end{tabular}

4 energy-intensive [1] - the power consumption can range from a few to several hundreds MW, depending on 5 the gas production and required pressures. The heating demand can be negligible if the feed has a high 6 gas-to-oil ratio, or amount to several tens, if the feed enters at low temperatures or is characterised by a 7 high viscosity. The cooling needs are usually much greater, as the produced gas should be cooled after each 8 compression operation.

These dissimilarities across fields result from differences in the (i) feed conditions (e.g. properties of the petroleum and water mixture); (ii) installed processes (e.g. treatment technologies, if relevant); (iii) operatand pressures) [2].

At present, floating production, storage and offloading (FPSO) facilities have gained more interest in countries such as Brazil. They may be operated on remote fields where an extensive pipeline infrastructure is not available for gas export, and the gas should either be injected or liquefied. They may be placed on fields where the produced petroleum contains large quantities of $\mathrm{CO}_{2}$, as high as $25 \%$ on a volume basis, and the produced gas must be treated accordingly with an absorption unit or a membrane process. They may be operating on fields with temperatures as low as $20^{\circ} \mathrm{C}$. In this case, an extensive network of oil heaters is required to reach the desired vapour pressure and to enhance the three-phase separation process. 
The priority of the oil and gas operators is to maximize the oil throughout. A major challenge when designing such systems is that the production of petroleum and water varies through the field lifetime. Usually, the production of crude oil ramps up in the first years, reaches a peak quickly, and then declines over time. Gas extraction follows a similar trend, but the gas production peak does not coincide with the oil production one. Water extraction usually increases over time steadily $[1,3,4]$.

According to the scientific literature, there exist several possibilities for improving the energy efficiency of offshore platforms [5]. For instance, energy integration is minimal, with possibly a couple of internal oil/oil or oil/water heat exchangers [6]. The use of waste heat from the gas turbine exhausts to generate additional power through Rankine cycles is not common, because of weight and space issues on offshore facilities. Centrifugal compressors are operating far from their nominal design points - gas is recirculated to prevent surge, which results in additional power consumption and cooling demand.

Previous works deal with the energy performance of these offshore facilities, with a focus on those present in the Gulf of Mexico, North and Norwegian Seas as well as the Brazilian pre-salt ones. Bothamley[1] compares the differences in processes between different facilities in various petroleum regions, and underlines that those depend on whether oil is to be exported for sales or for further treatment. Svalheim[3] emphasizes the high power demand of the gas compression and water injection modules over the whole field lifetime. A subsequent work [4] pinpoints that the facility may be operated in severe part-load conditions because of the large flow-rate variations.

The later researches of Voldsund et al. $[7,8]$ and of Nguyen et al. $[2,9,10]$ confirm these findings. Their works demonstrate that, for platforms processing high-temperature feeds, the heating demand is generally negligible. However, the compression operations represent the major share of the power consumption and exergy destruction. Similar results are found for ones with low-temperature feeds, with the difference that large amounts of exergy are also destroyed in the petroleum heating steps. Sánchez et al.[11] compare the thermodynamic performance of three FPSO operating modes and concludes that the energy requirements increase with the crude oil content in the feed. These studies show the dependency of the process performance on the feed conditions and properties, as well as on the operating modes. They pinpoint how the power demand and total exergy destruction on-site are related to the gas production. However, none actually investigates how different production profiles, together with different operating modes and feed compositions, actually impact the energy requirements of oil and gas platforms.

Gallo et al.[12] perform an exergy analysis of the gas compression systems on FPSOs and investigate the performance of these facilities over their lifetime, considering three different conditions. Their analysis takes intou account the part-load behaviour of these components. They find out that several equipment items, such as the compressors, are oversized for most of the operation time of the facility.

Finally, Riboldi and Nord[13,14] conduct a lifetime analysis of the offshore power generation system - they claim that offshore cogeneration of heat and power is a promising alternative to reduce the environmental 
impact of the oil and gas sector. However, the variability of these energy requirements creates challenges when designing the combined cycles, and the authors analyse and optimize different alternatives, based on the production profile of an oil field in Norway.

The aim of the present work is to assess the variations of the power, heating and cooling demands of oil and gas platforms over time, considering different feed properties, field conditions and operating modes. Based on a typical platform layout suggested by the industrial partners, the performance losses over the life cycle of the plant are analysed, and possible improvements of the processing and power plants are suggested.

\section{Methods}

\subsection{System description}

Oil and gas processing plants can be classified into two categories, depending on the degree of processing performed offshore and onshore, as stressed by Bothamley[1]. The plant can either produce a fully stabilized crude oil ready for sales, or an un-stabilized which requires further treatment onshore. This decision impacts the plant design, for example, the number of pressure levels of the separation train, the addition of an oil desalting process, the implementation of a gas treatment system, etc. However, despite these differences, several processes are found on all platforms (Figure 1):

- the production manifolds, in which the streams extracted from the wells are mixed and depressurized;

- the separation train, in which oil, gas and water are separated at descending pressures;

- the oil treatment process, in which oil is purified, stabilised, pumped and cooled to the desired storage/export pressure and temperature;

- the produced water treatment process, where the extracted water is cleaned from oil droplets before being injected back into the reservoir or released into the environment;

- the vapour recovery unit (VRU, or gas recompression), where the gas recovered from the separators is compressed to the initial feed pressure before further treatment;

- the gas compression and treatment process, where the produced gas is treated and compressed in several steps to the desired pressure; if the $\mathrm{CO}_{2}$-content of the recovered gas exceeds the desired specifications, membranes are implemented to decrease the $\mathrm{CO}_{2}$-fraction to less than $3 \%$ in the purified gas.

- the carbon dioxide compression process, where the $\mathrm{CO}_{2}$ separated from the natural gas is compressed for injection;

- the fuel gas system, where a fraction of the recovered gas is heated for further use in the gas turbines. 


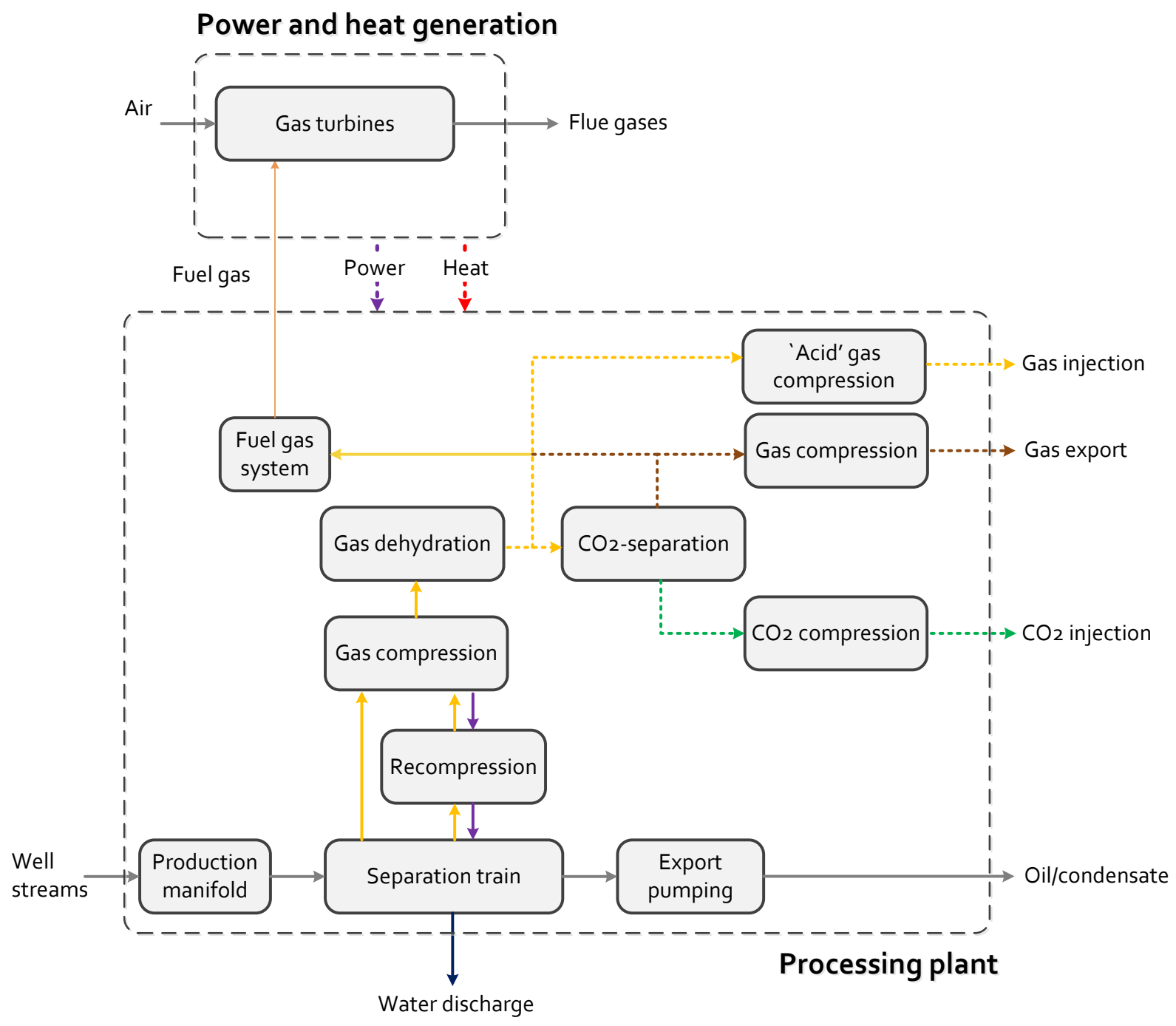

Figure 1: General overview of an offshore oil and gas platform

Power and heat are required on-site to drive the above-mentioned processes, and are generally generated by burning a fraction of the associated gas in gas turbines. In some cases, an additional furnace is implemented on-site for additional heating purposes, if the thermal exergy from the exhaust gases is not sufficient. It is thus possible to add in the analysis the two following processes:

- the gas turbines, where the actual power requirements are satisfied by burning the fuel gas;

- the waste heat recovery process, where a hot medium is used to recover heat from the exhaust gases for satisfying the heating demand in the petroleum separation. 
In the rest of this work, we refer to the first category of processes as the oil and gas processing plant, and the second category as the utilities.

\subsection{Case studies}

The present work builds on three feed compositions, three production profiles and three operating modes. The feed compositions taken for the analysis are the following:

- a gas condensate/near-critical oil (Composition 1), typical of Norwegian fields, with a negligible carbon dioxide content (less than 1\%), and about $81 \%$ methane, $6.5 \%$ ethane, $3.6 \%$ propane, $6 \%$ butanes, on a molar basis;

- a $\mathrm{CO}_{2}$-lean and volatile petroleum (Composition 2), from a Brazilian field, with a negligible carbon dioxide content (less than $1 \%$ ), and about $62 \%$ methane, $7 \%$ ethane, $5 \%$ propane and $3 \%$ butanes;

- a $\mathrm{CO}_{2}$-rich and volatile petroleum (Composition 3), from a Brazilian field, with a content of $26 \%$ carbon dioxide, $41 \%$ methane, $5 \%$ ethane and $3 \%$ propane.

These compositions are taken as a basis for the nominal operating point of the oil and gas facility, which corresponds to the peak production of oil. In other words, the feed contains a higher fraction of light hydrocarbons and carbon dioxide as the gas-to-oil ratio increases, and the oil treatment process is then operated in off-design conditions.

The production profiles selected for the simulations (Figure 2) are taken from the measurement database of the Norwegian Petroleum Directorate [15] - they correspond to actual fields operating in the last 30 years. The production profiles are selected for the simulations based on the similarities in terms of gas-to-oil ratios. For example, volatile oils are most often associated with high gas-to-oil ratios, at the opposite of heavy ones.


Figure 2: Oil, gas and water production and exports for the platform under study, from 1971 to 2015. 
The processing plant is run in different operational modes depending on the production rates of oil, gas and water. These modes are not common to all offshore platforms and differ from field to field - for example, several oil platforms do not have any gas export infrastructure, and gas is always injected into the reservoir. The present work considers three operation modes suggested by the project partners, which will likely be used in the development of future FPSOs.

At the beginning of the exploitation, and until the peaks of production of oil and gas are passed, the aim is to maximize the export of hydrocarbons (mode 1): all gas is exported to the shore. In parallel, water production continuously increases, and gas may be partly injected and partly exported (mode 2). The assumption presented in Sánchez et al.[11] is that this mode is activated as the water flow represents half of the liquid production. Finally, the gas production decreases until it is not enough for power production - it is then fully injected, and gas or diesel are imported and consumed (mode 3).

\subsection{System simulation}

The process models were developed with Aspen Plus version 7.2 [16] based on the Peng-Robinson [17] equation of state. The operating conditions and component efficiencies were set in the models based on the discussions with the industrial partners, using plant data and measurements. The most important assumptions are listed as follows, and the remaining ones are presented in Appendix A. A detailed overview of all state points is not given for confidentiality reasons.

- The processing plant was designed to handle a crude oil production of 150,000 barrels per day, as well as a gas production of $3,000,000 \mathrm{Sm}^{3} /$ day.

- The feed properties were taken to be $2,300 \mathrm{kPa}$ and $40{ }^{\circ} \mathrm{C}$ after gathering and depressurizing the well-streams in the production manifold.

- The following processes, dew point control with desiccants and oil desalting were not modelled in details, and were represented by a black-box model which output is set based on available measurements.

- Separation of carbon dioxide with membranes was modelled as a black-box with the initial $\mathrm{CO}_{2}$ concentration, feed and retentate pressures as inputs, and the flowrates and composition of each outflow as outputs. The model was calibrated and developed based both on the available data and the approach of Gassner et al.[18].

- Off-design behaviour of the heat exchangers was not modelled in details - it would, in practice, result in higher exergy destruction in the cooling system to maintain the same temperature approach in the heat exchangers. This increment is, however, negligible in comparison to the total amount of exergy destroyed on-site [6]. 
- The design point of the compressors was set to the maximum gas production encountered over the field lifetime. The discharge pressure of the centrifugal compressors was kept constant in the model in full and part-load conditions. The polytropic efficiency was fixed to $72 \%$ in all cases, while it would likely drop by 2 to $3 \%$-points in practice for a drop of the volume flow up to $40 \%$. The anti-surge system was set in the model to run when the inlet volume flow drops below $70 \%$ of the nominal one. These assumptions were based on the compressor maps provided by the project partners.

The details about the model and simulation assumptions are presented in Appendix A and Appendix B), they are based on industrial data provided by the project partners. The simulations were conducted for some specific years of the production profiles: the start- and end-life of the field, and the years in which the production of gas and oil reached a maximum.

\subsection{System performance}

The system performance is assessed by applying energy and exergy analyses. The first aim is to derive the variations of the heating, cooling and electricity demands as a function of the oil, gas and water profiles. The second goal is to calculate the corresponding exergy destruction and irreversibilities in the plant, for suggesting possible improvements. In the case of an offshore platform, energy enters and exits this system with material streams (e.g. petroleum feed, imported gas, fuel air, as well as oil, gas and produced water), with power (e.g. imported or exported electricity from the mainland or to other platforms) and with heat (e.g. heat losses by component radiation) (Equation 3).

$$
\begin{aligned}
& \dot{H}_{\text {feed }}+\dot{H}_{\mathrm{imp}}+\dot{H}_{\mathrm{air}}+\dot{H}_{\mathrm{cw}}+\dot{W}_{\mathrm{imp}}=\sum_{k} \dot{H}_{k}+\dot{H}_{\mathrm{exh}}+\dot{H}_{\mathrm{rw}}+\dot{W}_{\mathrm{exp}} \\
& \dot{H}_{\mathrm{feed}}+\dot{W}_{\mathrm{UT}}+\dot{Q}_{\mathrm{UT}, \text { heat }}=\sum_{k} \dot{H}_{k}+\dot{Q}_{\mathrm{PP}, \mathrm{cool}} \\
& \dot{H}_{\mathrm{k}, \text { fuel }}+\dot{H}_{\mathrm{air}}=\dot{Q}_{\mathrm{UT}, \mathrm{cool}}+\dot{Q}_{\mathrm{UT}, \text { heat }}+\dot{W}_{\mathrm{UT}}
\end{aligned}
$$

where, on a rate basis:

- $\dot{H}$ stands for the enthalpy carried with the ingoing material flows (feed denoting the feed streams from the wells, imp the imported gas for injection or power generation, air for the air processed through the gas turbines);

- or for the outgoing streams (cw for the seawater used for cooling needs, exh for the exhaust gases, rw for the treated and rejected cooling water, and $\mathrm{k}$ for the several oil and gas streams);

- $\dot{W}$ for the energy transferred with power, imported or exported to the mainland or other platforms. 
- $\dot{W}_{\mathrm{UT}}$ is the power consumed within the separation and treatment modules, as well as in electric heaters, which is produced in the utility plant;

- $\dot{Q}_{\mathrm{PP}, \text { heat }}$ is the heat entering the processing plant, generally by direct heat exchange with the exhausts of a gas turbine, or by indirect heat exchange, by using a heating medium (e.g. hot water or hot glycol);

- $\dot{Q}_{\mathrm{PP}, \text { cool }}$ is the heat entering the processing plant, generally by direct heat exchange with the exhausts of a gas turbine, or by indirect heat exchange, by using a heating medium (e.g. hot water or hot glycol);

- $\dot{Q}_{\mathrm{UT}, \text { cool }}$ is the energy transferred from the power plant to the cooling medium (e.g. cooling air, seawater or glycol-water mixtures) in, for instance, a steam condenser.

As underlined by the First Law of Thermodynamics, energy cannot be created or destroyed, but can only transformed from one form to another. An energy mapping shows where energy is converted (for example from heat to electricity) and dissipated (for instance when discharging water into the sea), but cannot be used consistently to assess the system performance.

Exergy may be defined as 'the maximum theoretical useful work (shaft work or electrical work) as the system is brought into complete thermodynamic equilibrium with the thermodynamic environment while the system interacts with it only' [19]. Unlike energy, exergy is not conserved but is destroyed in real systems because of irreversible phenomena, such as heat transfer across finite temperature differences and pressure drops. This concept can be therefore used to pinpoint, identify and quantify performance losses (6):

$$
\begin{aligned}
& \dot{E}_{\mathrm{feed}}+\dot{E}_{\mathrm{imp}}+\dot{E}_{\mathrm{air}}+\dot{E}_{\mathrm{cw}}+\dot{E}_{\mathrm{imp}}^{W}=\sum_{k} \dot{E}_{k}+\dot{E}_{\mathrm{exh}}+\dot{E}_{\mathrm{rw}}+\dot{E}_{\mathrm{exp}}^{W}+\dot{E}_{\mathrm{d}, \mathrm{OP}} \\
& \dot{E}_{\mathrm{feed}}+\dot{E}_{\mathrm{UT}}^{W}+\dot{E}_{\mathrm{UT}, \text { heat }}^{Q}=\sum_{k} \dot{E}_{k}+\dot{E}_{\mathrm{PP}, \mathrm{cool}}^{Q}+\dot{E}_{\mathrm{d}, \mathrm{PP}} \\
& \dot{E}_{\mathrm{k}, \text { fuel }}+\dot{E}_{\mathrm{air}}=\dot{E}_{\mathrm{UT}, \mathrm{cool}}^{Q}+\dot{E}_{\mathrm{UT}, \text { heat }}^{Q}+\dot{E}_{\mathrm{UT}}^{W}+\dot{E}_{\mathrm{exh}}
\end{aligned}
$$

where, on a rate basis:

- $\dot{E}$ denotes the exergy flow associated with a given stream of matter;

- $\dot{E}^{W}$ denotes the exergy transferred with power, and has the same value than its energy;

- $\dot{E}^{Q}$ denotes the exergy transferred with heat, and has a smaller value than its energy, as it depends on the temperatures of the environment and at which the heat transfer takes place;

- $\dot{E}_{\mathrm{d}}$ is the exergy destroyed in the overall (OP), processing (PP) and utility plants (UP). 
The concept of exergy is intrinsically linked to a dead state, which was defined here as $1.013 \mathrm{bar}, 28^{\circ} \mathrm{C}$, and with the chemical composition of the environment defined by Morris and Szargut[20]. The chemical exergy of crude oil was calculated using the correlations of Rivero et al.[21].

\subsection{Performance indicators}

The following indicators are defined to assess the efficiency of each process and the overall system performance:

- the specific power consumption per unit of exported oil and gas $w$, if relevant;

- the specific energy demand, which considers then both the power and heating needs, also called energy intensity in the literature [22];

- the specific exergy demand, i.e. the exergy required to drive the overall system, on the process side;

- the exergy destruction ratio, defined as the exergy destruction taking place in a component or process over the exergy destruction of the entire system.

These indicators are given on an oil equivalent (o.e.) basis, meaning that $1 \mathrm{Sm}^{3}$ of oil is equivalent to $1000 \mathrm{Sm}^{3}$ of gas.

\section{Results}

\subsection{Energy assessment}

Although the feed compositions investigated in the present work (Figure 3, Figure 4 and Figure 5) present significant differences in carbon dioxide, methane and heavy hydrocarbons, several general trends can be drawn.

First, the heating demand is associated with two main processes, namely the oil and gas separation, and the fuel gas heating. The first process is the most energy-intensive, as oil, gas and water are heated between the first and second stage to enhance phase separation. The heating demand reaches a maximum at the oil peak production - the variations of the gas and water flows have little impact, as most gas and water are recovered upstream. The heat requirements of the second process increase together with the gas production, since more fuel gas needs to be preheated with greater power demand. The heat demands of the other processes, such as the dew point control or gas dehydration, are negligible in comparison.

Secondly, the cooling demand is greater than the heating requirements, except for cases with very low gas-to-oil ratios (heavy oils). It usually corresponds to compressor after-cooling, with heat discharged at an initial temperature of $100-200{ }^{\circ} \mathrm{C}$, or to condensate scrubbing, with heat released at around $80^{\circ} \mathrm{C}$. The peak demand is reached when the gas production attains its maximum. 


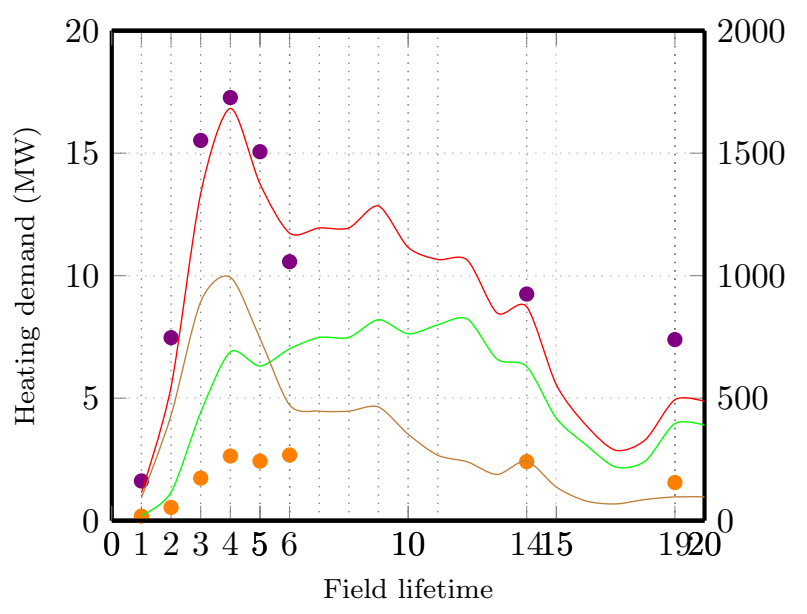

- Fuel gas $\bullet$ Separation

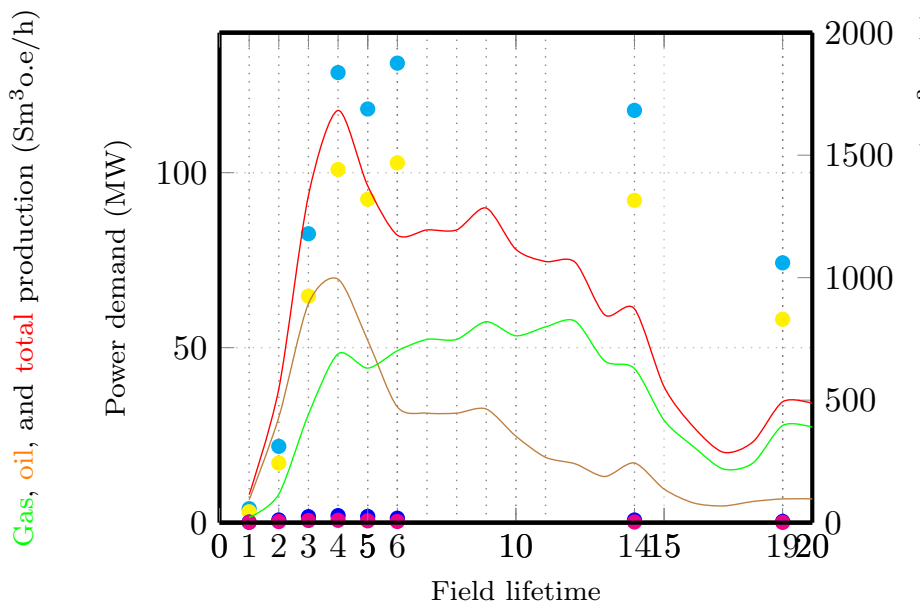

- Gas compression Gas treatment $\bullet$ VRU • Oil treatment

Figure 3: Power and heating demands, sorted by sub-system, for the Norwegian petroleum case, on an energy basis
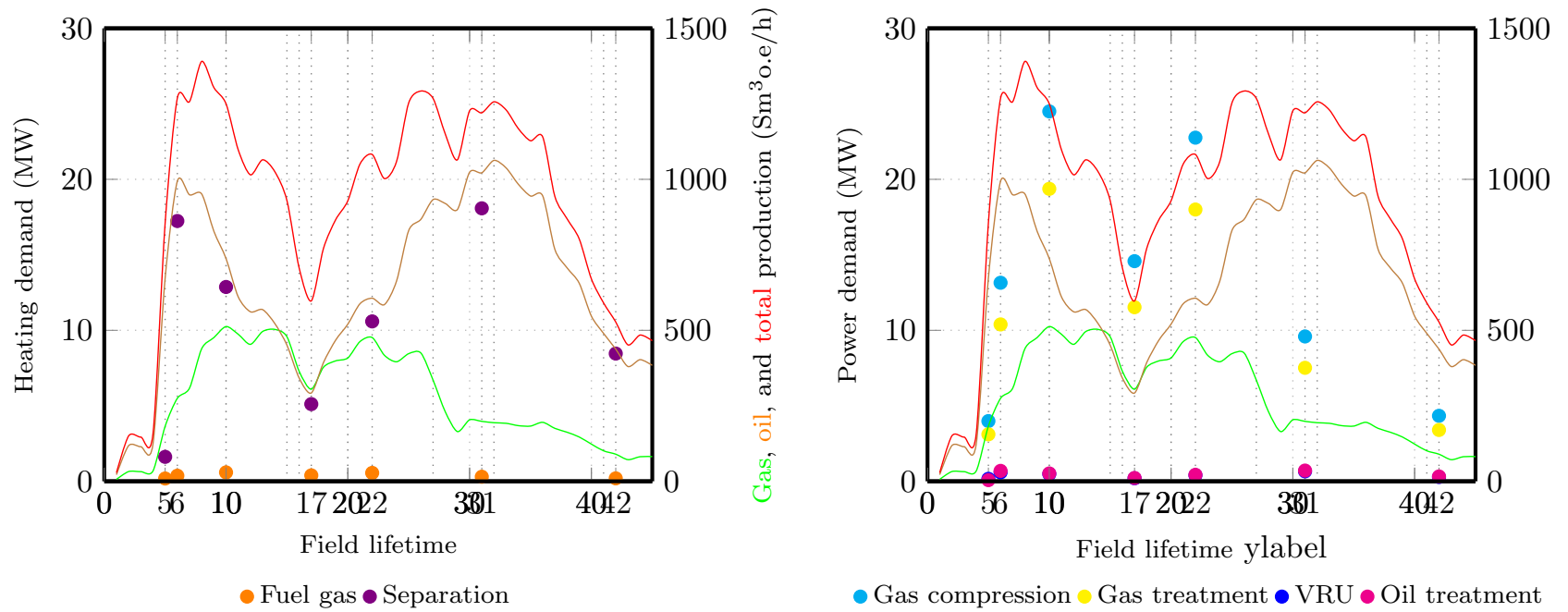

Figure 4: Power and heating demands, sorted by sub-system, for the volatile case, on an energy basis

Finally, the net power consumption is highly dependent on the gas production rate, whether the gas has a high or low $\mathrm{CO}_{2}$-content, but is moderately sensitive to the oil production. The gas compression and treatment processes represent the major share of the total power demand $(\geq 80 \%)$.

For petroleum with high $\mathrm{CO}_{2}$-content, the use of membranes to purify the incoming gas stream has a significant impact on the total power demand, since the permeate is recovered at 3 bar, and must be re-compressed up to 55 bar. In that case, the total power demand can be roughly divided into $1 / 3$ for the gas compression, $1 / 3$ for the gas treatment, $1 / 3$ for the $\mathrm{CO}_{2}$-compression and injection. The switch from one mode of operation to another - from the gas export to the gas injection mode - results in lower power 

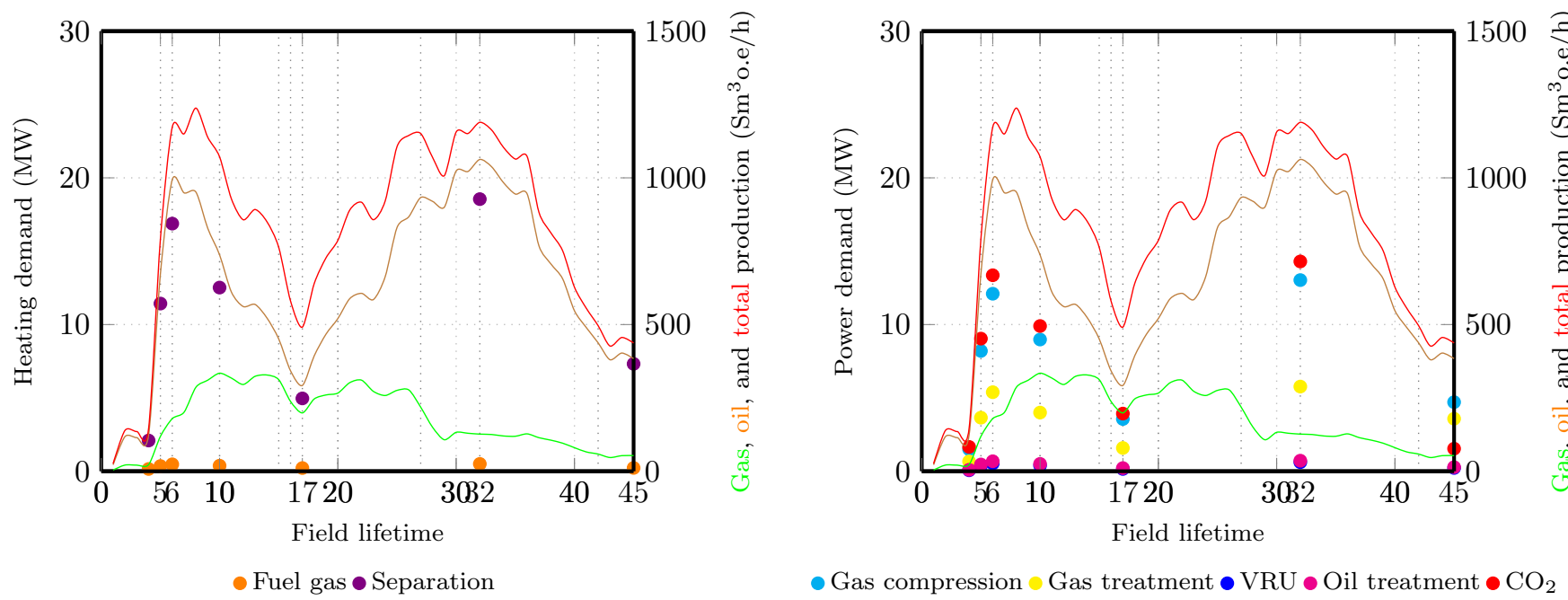

Figure 5: Power and heating demands, sorted by sub-system, for the volatile case with $\mathrm{CO}_{2}$, on an energy basis

consumption on-site for the two following reasons. First, carbon dioxide is not separated from methane and is thus directly compressed from 55 bar instead of 3 bar. Secondly, the injection pressure is lower for acid gas mixtures than for nearly-pure carbon dioxide.

\subsection{Exergy analysis}

The exergy accounting shows that the exergy inflowing and outflowing the oil and gas platform is dominated by the chemical exergy of the hydrocarbon streams at all phases of the petroleum production (Figure 6 , Figure 7, Figure 8). The physical exergy of these streams is negligible in comparison, representing less than $2 \%$ of the feed exergy. Similarly, the exergy of the other material streams, e.g. air and exhaust gases, is minor, and the exergy destroyed on-site represents less than $3 \%$ of the feed exergy.

Very little exergy is actually transformed and consumed on-site, most only transits from the inlet to the outlets and is carried with the hydrocarbon and carbon dioxide streams. It is consumed as power and heat, generated from the combustion of fuel gas, to separate and purify the crude oil mixture into its gas, oil and water phases. Few differences can be depicted between the peak and end-life phases, which are characterised by two different modes of operation. In the first one, natural gas is purified and exported, while, in the second case, acid gas is not purified and is directly injected.

A more detailed analysis of the exergy destruction on-site (Figure 9, Figure 10 and Figure 11) shows that the gas turbines represent the main share of the total irreversibilities on-site, because of the combustion process. They represent up to $60-65 \%$ over time. The processes in the processing plant that display the largest exergy destruction are the gas compression and treatment processes, which are also the greatest power consumers. The membranes and vapour recovery unit follow in case that carbon dioxide must be 


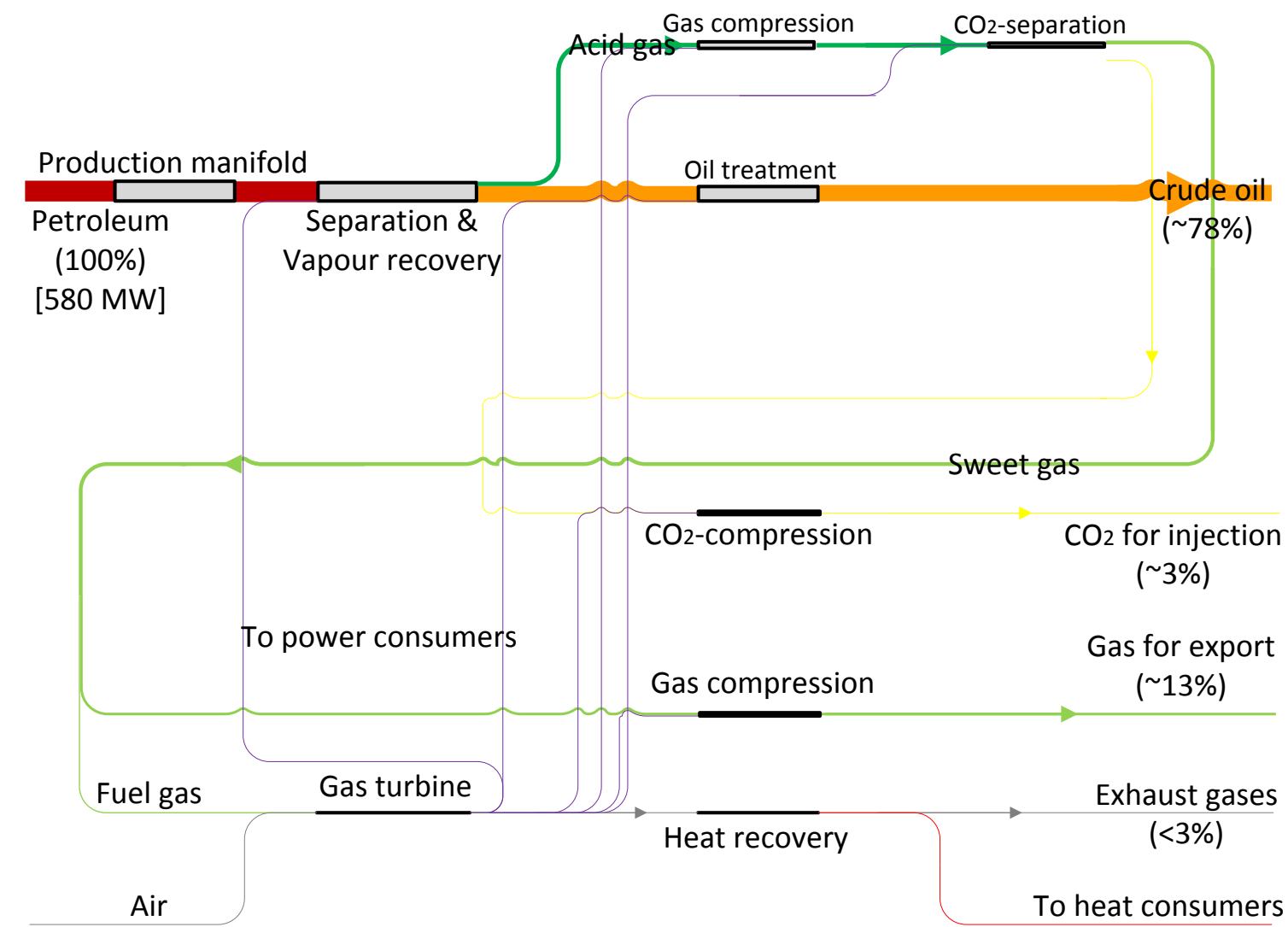

Figure 6: Grassmann diagram of an offshore oil and gas platform for a feed with high $\mathrm{CO}_{2}$-composition, at the beginning of the hydrocarbon production

recovered, because of the large pressure drop across the membrane in the first process and the high pressure ratio in the second.

The exergy flow associated with the heat exchanges on the offshore platform represents up to 15-20\% of the associated energy flow. Exergy is destroyed in the heat recovery system because of the large temperature gap between the exhaust gases $\left(400\right.$ to $\left.600^{\circ} \mathrm{C}\right)$ and the petroleum $\left(\simeq 40-80^{\circ} \mathrm{C}\right)$. It is also lost when discharged into the environment with the flue gases at the outlet of the heating system $\left(300\right.$ to $\left.500{ }^{\circ} \mathrm{C}\right)$.

A comparison with the other case studies shows that the exergy demands are well-correlated with the gas production profiles (power) and oil (heat), which suggests that these trends can be generalized to all feed compositions. However, it is not possible to derive a direct relation between the total oil and gas production (on an oil equivalent basis) and the energy demands. In the analyzed scenarios, there is no supplementary burning of fuel gas to supply the required thermal load, and the trends derived in this work may not be 


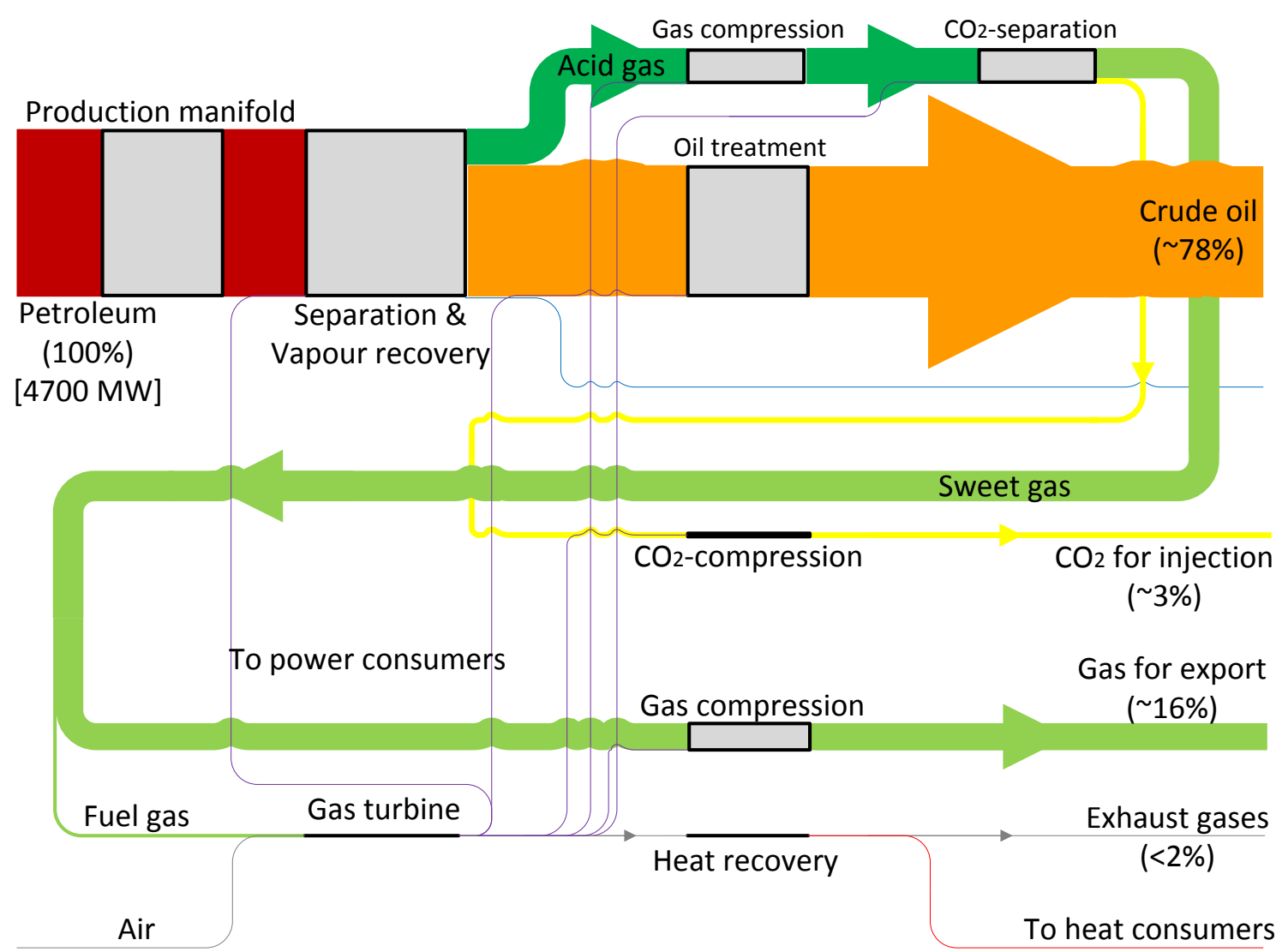

Figure 7: Grassmann diagram of an offshore oil and gas platform for a feed with high $\mathrm{CO}_{2}$-composition, at the peak of the oil production

fully applicable to such cases.

These results show the importance of focusing improvement efforts on the compression processes, as they represent the greatest share of exergy destruction and power consumption in all cases, at all life stages. As mentioned in the literature in this field [12], gas compressors are generally oversized on offshore platforms, which implies that they run far from their nominal design points over the field lifetime. For operational matters, gas is recycled (anti-surge) to sustain a minimal gas flow, meaning that power is consumed and exergy is destroyed without any useful purpose, in exergetic terms. Any improvement of this system would result in smaller power consumption, and in turn in smaller fuel demand and lower exergy destruction in the power generation system.

Efforts should as well focus on the design of the utility plant. Gas turbines and cogeneration plants offshore are usually designed for the peak production of hydrocarbons, but it is critical to assess the variability 


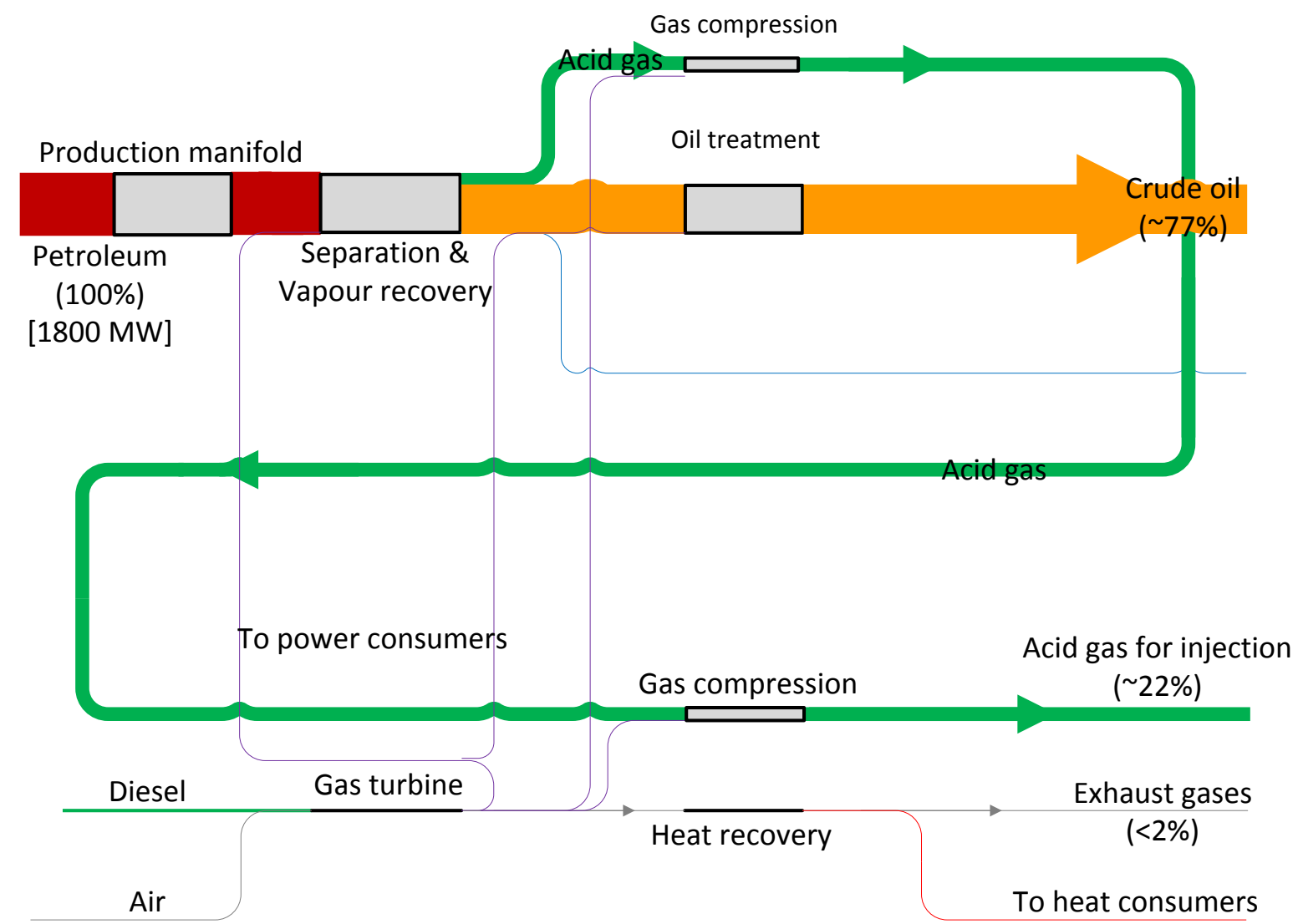

Figure 8: Grassmann diagram of an offshore oil and gas platform for a feed with high $\mathrm{CO}_{2}$-composition, at the end-life of the oil production

cas

\subsection{Performance indicators}

The comparison of the performance indicators for the three compositions under study (Table 1, Table 2 and Table 3), considering the field lifetime, shows the following trends. Firstly, the specific power consumption, energy intensity and specific exergy demand increase with time. The high values reached at the end of the field lifetime illustrate that large amounts of power are required to drive the gas compression and injection systems - although small quantities of gas are extracted, the flows into the compressors are nearly constant as gas is recirculated to prevent surge issues. 




Figure 9: Distribution of the exergy destruction per process for the gas condensate case

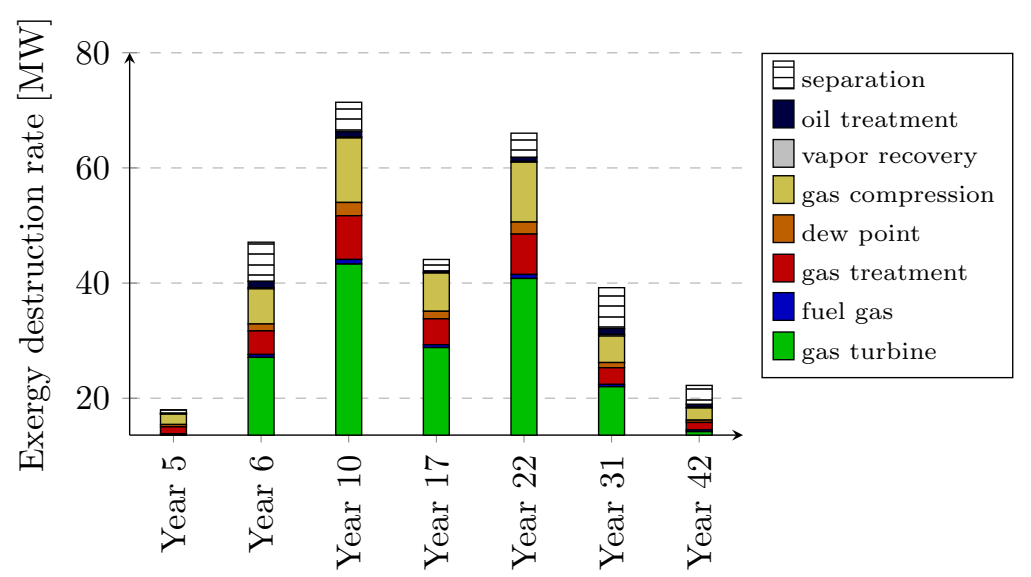

Figure 10: Distribution of the exergy destruction per process for the volatile case

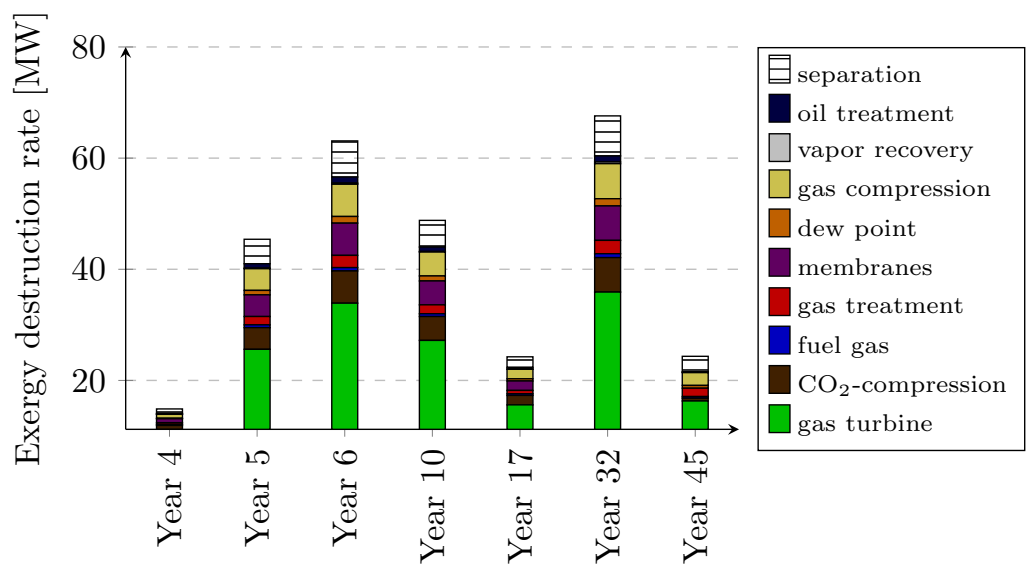

Figure 11: Distribution of the exergy destruction per process for the $\mathrm{CO}_{2}$-rich petroleum case 
Table 1: Performance indicators of the offshore platform for the gas condensate case

\begin{tabular}{lrrrrrrrr}
\hline & 1 & 2 & 3 & 4 & 5 & 6 & 14 & 19 \\
\hline Oil production $\left(\frac{\mathrm{Sm}^{3}}{\mathrm{hr}}\right)$ & 92.8 & 427.9 & 891.3 & 993.0 & 744.9 & 475.5 & 247.5 & 99.8 \\
Hydrocarbon production $\left(\frac{\mathrm{Sm}_{\text {o.e. }}^{3}}{\mathrm{hr}}\right)$ & 134.2 & 662.7 & 1788.4 & 2393.0 & 2026.1 & 1900.3 & 1525.1 & 904.2 \\
Power consumption $\left(\frac{\mathrm{MJ}}{\mathrm{Sm}_{\text {o.e. }}^{3}}\right)$ & 197.0 & 216.8 & 301.1 & 349.3 & 378.3 & 446.5 & 497.7 & 528.6 \\
Power and heat use $\left(\frac{\mathrm{MJ}}{\mathrm{Sm}_{\text {o.e. }}^{3}}\right)$ & 264.6 & 281.7 & 366.5 & 415.2 & 448.3 & 518.0 & 577.4 & 619.7 \\
Exergy demand $\left(\frac{\mathrm{MJ}}{\mathrm{Sm}_{\text {o.e. }}^{3}}\right)$ & 203.4 & 222.6 & 305.8 & 353.4 & 382.4 & 449.9 & 501.7 & 533.8 \\
\hline
\end{tabular}

Secondly, there are no major differences between the values of the specific power consumption and specific exergy demand. The heating demand is, on an energy basis, smaller than the power demand, and is at low to moderate temperatures, meaning that the corresponding exergy demand is small as well.

Table 2: Performance indicators of the offshore platform for the volatile case

\begin{tabular}{lrrrrrrr}
\hline & 9 & 10 & 14 & 21 & 26 & 35 & 46 \\
\hline Oil production $\left(\frac{\mathrm{Sm}^{3}}{\mathrm{hr}}\right)$ & 92.8 & 993.7 & 738.8 & 293.1 & 607.4 & 1018.7 & 436.5 \\
Hydrocarbon production $\left(\frac{\mathrm{Sm}_{\text {o.e. }}^{3}}{\mathrm{hr}}\right)$ & 134.2 & 1135.0 & 1003.7 & 450.0 & 853.4 & 1121.0 & 481.7 \\
Power consumption $\left(\frac{\mathrm{MJ}}{\mathrm{Sm}_{\text {o.e. }}^{3}}\right)$ & 197.0 & 78.7 & 160.9 & 212.2 & 175.4 & 59.3 & 62.3 \\
Power and heat use $\left(\frac{\mathrm{MJ}}{\mathrm{Sm}_{\text {o.e. }}^{3}}\right)$ & 264.6 & 142.4 & 225.6 & 278.0 & 240.3 & 124.2 & 133.2 \\
Exergy demand $\left(\frac{\mathrm{MJ}}{\mathrm{Sm}_{\text {o.e. }}^{3}}\right)$ & 203.4 & 86.0 & 167.3 & 218.0 & 181.6 & 67.1 & 70.7 \\
\hline
\end{tabular}

Finally, the specific power and exergy demands are far higher when processing gas condensate than volatile petroleum, because larger gas flowrates need to be processed. These findings suggest therefore that the power demand on offshore platforms is always strongly correlated to the gas production and little to the oil extraction.

Table 3: Performance indicators of the offshore platform for the $\mathrm{CO}_{2}$-rich petroleum case

\begin{tabular}{lrrrrrrr}
\hline & 4 & 5 & 6 & 10 & 17 & 32 & 45 \\
\hline Oil production $\left(\frac{\mathrm{Sm}^{3}}{\mathrm{hr}}\right)$ & 123.3 & 673.3 & 993.7 & 737.5 & 292.4 & 1041.7 & 376.3 \\
Hydrocarbon production $\left(\frac{\mathrm{Sm}_{\text {o.e. }}^{3}}{\mathrm{hr}}\right)$ & 129.7 & 717.0 & 1059.2 & 785.6 & 310.2 & 1112.2 & 376.3 \\
Power consumption $\left(\frac{\mathrm{MJ}}{\mathrm{Sm}_{\text {o.e. }}^{3}}\right)$ & 110.9 & 109.0 & 109.0 & 108.9 & 109.6 & 111.5 & 98.9 \\
Power and heat use $\left(\frac{\mathrm{MJ}}{\mathrm{Sm}_{\text {o.e. }}^{3}}\right)$ & 183.1 & 177.9 & 177.6 & 177.7 & 179.5 & 183.0 & 180.1 \\
Exergy demand $\left(\frac{\mathrm{MJ}}{\mathrm{Sm}_{\text {o.e. }}^{3}}\right)$ & 119.0 & 116.7 & 116.7 & 116.7 & 117.5 & 119.5 & 108.2 \\
\hline
\end{tabular}




\section{Discussion}

The scientific literature on the performance of oil and gas platforms deals with the modelling and assessment of existing case studies (e.g. Norwegian and Brazilian plants). The work of Oliveira Jr. and Van Hombeeck[23] deals with the exergy performance of a Brazilian facility, and is complemented by the research of Voldsund et al.[8], where the sources of exergy destruction and losses on four offshore facilities, located in the Norwegian and North Seas, are analysed. The studies of Nguyen et al.[2,10] deal with the lifetime performance of one of these platforms, analysing how the variations of oil and gas affect the power, heating and cooling demands. The present work adopts a similar approach, by analysing different production profiles and feed compositions. The corresponding results are in accordance with the findings presented in the literature, although all case studies are very different. They stress the large power demand and exergy destruction over the field lifetime of the gas compression and injection units, in all cases. These works demonstrate therefore how critical it is to design and operate efficiently gas compression systems, in whichever cases, to enhance the lifetime performance of oil and gas platforms. This conclusion justifies the need for further work on these processes, proposing improvements, as done in Gallo et al.[12].

In addition, other works focus on the design and optimization of the utility system (gas turbine and heat recovery) - changes of the processing plant may be hard to achieve, as the system design and operating parameters are constrained to maximise the oil and gas recoveries. Improving the efficiency of these processes is also a way to reduce the fuel use on-site and the resulting $\mathrm{CO}_{2}$-emissions. As emphasised in Riboldi and Nord[13], the challenges for implementing an efficient combined cycle are related to the variability of the power-to-heat ratio and the variations of the power and heating demands. One of the main findings is that there is no generic system layout that can be implemented to ensure a high efficiency of the combined cycle over time. For example, backpressure steam turbine cycles, although feasible in all the assessed cases, are attractive only if the facility has large heat demands at low temperatures. Extraction steam turbine cycles are preferred if the heat requirements are limited. Organic Rankine cycles may be a possibility, as discussed in Pierobon et al.[24], but the studies on how to integrate efficiently these systems is missing.

These works, although different in scope, present similar conclusions: they pinpoint the gas compression and waste heat recovery units as the units to focus on and improve. However, the ways to increase their efficiency differ with the field, production rates and energy demands. Future work should therefore focus on developing a robust modelling and optimization approach which accounts for these parameters, in order to design appropriately offshore platforms.

\section{Conclusion}

The life performance of oil and gas facilities was assessed by modelling these plants with the software Aspen Plus, based on three feed compositions with different methane, carbon dioxide and heavy hydro- 
carbons contents. Two production profiles and three operation modes were considered. The energy and exergy analysis highlight the strong correlation of the power demand with the gas production, and of the heating demand with the oil extraction. The compression processes represent the major share of the total energy use and exergy destruction $(\geq 80 \%)$ in the processing plant. A high content of carbon dioxide in the feed slightly changes the overall picture, as large quantities of exergy are destroyed in the membrane when recovering and recompressing the permeate stream. The gas turbines represent a large share of exergy destruction ( $\geq 60-65 \%)$ and losses because of the combustion process and exhaust losses. These findings are valid for all cases at all life stages, and suggest focusing improvement efforts on the compression operations and waste heat recovery possibilities. In practice, the exergy destruction expected in those processes is likely to be even greater, as gas turbines and compressors operating in off-design conditions face severe drops in efficiency.

\section{Acknowledgements}

The present work was supported by the National Agency of Petroleum, Natural Gas and Biofuels (ANP) and Shell Brasil, through Investment in Research, Development and Innovation Clause, contained in contracts for Exploration, Development and Production of Oil and Natural Gas. The authors acknowledge the financial support from the Fundação de Amparo à Pesquisa do Estado de São Paulo (São Paulo research foundation, FAPESP), through the grant 2015/09157-1, and from the Conselho Nacional de Desenvolvimento Científico e Tecnológico (Brazilian National Council for Scientific and Technological Development, CNPq), through the grant 304935/2016-6.

\section{References}

[1] Bothamley M. Offshore Processing Options for Oil Platforms. In: Proceedings of the SPE Annual Technical Conference and Exhibition. Houston, USA: Society of Petroleum Engineers; 2004, p. 1-17 (Paper SPE 90325).

[2] Nguyen TV, Fülöp T, Breuhaus P, Elmegaard B. Life performance of oil and gas offshore platforms -site-scale integration and exergy-based assessment. Energy 2014;73:282-301.

[3] Svalheim SM. Environmental Regulations and Measures on the Norwegian Continental Shelf. In: Proceedings of the SPE International Conference on Health, Safety and Environment in Oil and Gas Exploration and Production. Kuala Lumpur, Malaysia: Society of Petroleum Engineers; 2002, p. 1-10 (Paper SPE 73982).

[4] Svalheim SM, King DC. Life of Field Energy Performance. In: Proceedings of the SPE Offshore Europe Conference. July; Aberdeen, United Kingdom: Society of Petroleum Engineers; 2003, p. 1-10 (Paper SPE 83993).

[5] Nguyen TV, Voldsund M, Breuhaus P, Elmegaard B. Energy efficiency measures for offshore oil and gas platforms. Energy $2016 ; 117: 325-40$.

[6] Nguyen TV, Tock L, Maréchal F, Elmegaard B. Oil and gas platforms with steam bottoming cycles: Retrofit integration and thermo-environomic evaluation. Applied Energy 2014;131:222-37.

[7] Voldsund M, Ertesvåg IS, He W, Kjelstrup S. Exergy Analysis of the Oil and Gas Processing a Real Production Day on a North Sea Oil Platform. Energy 2013;55:716-27. 
[8] Voldsund M, Nguyen TV, Elmegaard B, Ertesvåg I, Røsjorde A, Jøssang K, et al. Exergy destruction and losses on four North Sea offshore platforms: A comparative study of the oil and gas processing plants. Energy 2014;74:45-58.

[9] Nguyen TV, Pierobon L, Elmegaard B, Haglind F, Breuhaus P, Voldsund M. Exergetic assessment of energy systems on North Sea oil and gas platforms. Energy 2013;62:23-36.

[10] Nguyen TV, Jacyno T, Breuhaus P, Voldsund M, Elmegaard B. Thermodynamic analysis of an upstream petroleum plant operated on a mature field. Energy 2014;68:454-69.

[11] Sánchez YAC, de Oliveira Júnior S, da Silva JAM, Nguyen TV. Energy and Exergy Performance of three FPSO Operational Modes. In: Proceedings of the 23rd ABCM International Congress of Mechanical Engineering. 2015,.

[12] Gallo WL, Gallego AG, Acevedo VL, Dias R, Ortiz HY, Valente BA. Exergy analysis of the compression systems and its prime movers for a fpso unit. Journal of Natural Gas Science and Engineering 2017;44:287-98.

[13] Riboldi L, Nord LO. Lifetime assessment of combined cycles for cogeneration of power and heat in offshore oil and gas installations. Energies 2017;10(6):744.

[14] Riboldi L, Nord LO. Concepts for lifetime efficient supply of power and heat to offshore installations in the north sea. Energy Conversion and Management 2017;148:860-75.

[15] Norwegian Petroleum Directorate. Standards relating to measurement of petroleum for fiscal purposes and for calculation of $\mathrm{CO}_{2}$-tax. Tech. Rep.; Norwegian Petroleum Directorate; 2012.

[16] Aspen Technology . Aspen Plus - Modelling Petroleum Processes. Burlington, USA: Aspen Technology; 1999.

[17] Peng DY, Robinson DB. A New Two-Constant Equation of State. Industrial \& Engineering Chemistry Fundamentals $1976 ; 15(1): 59-64$.

[18] Gassner M, Baciocchi R, Maréchal F, Mazzotti M. Integrated design of a gas separation system for the upgrade of crude sng with membranes. Chemical Engineering and Processing: Process Intensification 2009;48(9):1391-404.

[19] Bejan A, Tsatsaronis G, Moran M. Thermal Design \& Optimization. New York, USA: John Wiley \& Sons; 1996.

[20] Morris DR, Szargut J. Standard chemical exergy of some elements and compounds on the planet Earth. Energy $1986 ; 11(8): 733-55$.

[21] Rivero R, Rendon C, Monroy L. The Exergy of Crude Oil Mixtures and Petroleum Fractions: Calculation and Application. International Journal of Applied Thermodynamics 1999;2(3):115-23.

[22] Voldsund M, Nguyen TV, Elmegaard B, Ertesvåg IS, Røsjorde A, He W, et al. Performance indicators for evaluation of North Sea oil and gas platforms. In: Proceedings of ECOS 2013 - The 26th International Conference on Efficiency, Cost, Optimization, Simulation and Environmental Impact of Energy Systems. 2013,.

[23] Oliveira Jr. SD, Van Hombeeck M. Exergy Analysis of Petroleum Separation Processes in Offshore Platforms. Energy Conversion and Management 1997;38(15-17):1577-84.

[24] Pierobon L, Nguyen TV, Larsen U, Haglind F, Elmegaard B. Multi-objective optimization of organic rankine cycles for waste heat recovery: Application in an offshore platform. Energy 2013;58:538 -49.

[25] Nguyen TV, de Oliveira Júnior S. System evaluation of offshore platforms with gas liquefaction processes. Energy 2018;144:594-606.

[26] McCarrick MT, Kenneth M. LM2500 to LM2500+DLE Gas Turbine Combined Cycle Plant Repowering. GE Energy; 2012.

The present appendix presents the modelling and simulation data as given in Nguyen and de Oliveira Júnior[25], which is repeated here to ease reading. 


\section{Appendix A. Processing plant modelling}

The reservoir fluids from the producing wells enter the production manifolds, where all streams are mixed and depressurised to about $1500 \mathrm{kPa}$. They then enter the separation section, in which oil, gas and water are separated in several stages operated at different temperature and pressure levels. The pressure is decreased at the inlet of each separator and the temperature may be increased to enhance the separation between the water and hydrocarbon phases. Oil is further treated, and the water and salt contents are decreased to satisfy the crude export requirements. The produced water is discharged into the sea or in a dedicated reservoir after removal of the oil droplets. The assumptions on the component models are presented in Table A.4 (e.g. efficiencies) and the process pressures and temperatures are given in Table A.5.

Table A.4: Component parameters of the offshore oil and gas processing

\begin{tabular}{ll}
\hline Parameter & Value \\
\hline Heat losses & - \\
Pressure drops & - \\
Oil cooler temperatures & $60{ }^{\circ} \mathrm{C}$ \\
Gas cooler temperatures & $40{ }^{\circ} \mathrm{C}$ \\
Compressor efficiency & $72 \%$ \\
Generator efficiency & $96 \%$ \\
\hline
\end{tabular}

Table A.5: Operating conditions of the feed processing

\begin{tabular}{lll}
\hline Process & Parameter & Value \\
\hline Separation & Pressure & $\geq 1.5 \mathrm{bar}$ \\
& & $\leq 15 \mathrm{bar}$ \\
& Temperature & $85^{\circ} \mathrm{C}$ \\
Oil export & Reid Vapor & $0.7 \mathrm{bar}$ \\
& Pressure $\left(38^{\circ} \mathrm{C}\right)$ & \\
Vapor recovery unit & Pressures & $\geq 1.5 \mathrm{bar}$ \\
& & $\leq 15 \mathrm{bar}$ \\
Gas compression & Pressures & $\geq 15 \mathrm{bar}$ \\
& & $\leq 55 \mathrm{bar}$ \\
\hline
\end{tabular}

As a basis for the simulations, the reservoir fluid enters at $20^{\circ} \mathrm{C}$ and $1500 \mathrm{kPa}$, in a three-phase equilibrium - gas, oil and water phases (vapor and two non-miscible liquids). These feed conditions correspond to real-time data and to assumptions presented in the literature [23]. The gas recovered from the second 
and third separation stages is first compressed in a vapour recovery unit to the initial feed pressure. The number of recompression stages is equal or higher to the number of separation levels. The recompressed gas is then mixed with the gas recovered at the first stage and compressed further to $5500 \mathrm{kPa}$ for treatment purposes (Table A.6). High contents of carbon dioxide when exporting gas are not desirable because of possible corrosion issues. Membrane separation is the preferred system on Brazilian platforms, considering a difference of 55 to 3 bar between the feed and the retentate.

Table A.6: Specifications of the gas treatment technologies

\begin{tabular}{lll}
\hline Process & Parameter & Value \\
\hline Dew point control & Pressure & $\geq 50 \mathrm{bar}$ \\
Membranes & $\mathrm{CO}_{2}$-fraction & $3 \%$ \\
& Selectivity $\mathrm{CO}_{2} / \mathrm{CH}_{4}$ & 21 \\
& Permeability & 9 barrer \\
& Permeate pressure & $3 \mathrm{bar}$ \\
\hline
\end{tabular}

The choice of purifying, injecting or exporting gas depends on the initial petroleum composition (Table A.7). According to FPSO operators, gas should be exported at the first stage of a field lifetime, when the ratio oil - gas to water is at its maximum, and the hydrocarbon production is near its peak. In the declining phase, when the crude oil contains about $50 \%$ of water, part of the gas may still be treated and exported, while the remaining fraction is directly injected. Finally, in end-life conditions, when the water and carbon dioxide contents represent more than 80-90\% of the total feed content, the acid gas is directly injected, and clean gas is imported from other facilities to fuel the gas turbines (Table A.7).

Table A.7: Specifications of the final gas processing operations

\begin{tabular}{lll}
\hline Process & Parameter & Value \\
\hline Gas lift & Pressure & $\leq 250 \mathrm{bar}$ \\
Gas injection & Acid gas pressure & $\leq 450 \mathrm{bar}$ \\
& Clean gas pressure & $\leq 550 \mathrm{bar}$ \\
Gas export & Pressure & $\leq 245 \mathrm{bar}$ \\
\hline
\end{tabular}

\section{Appendix B. Utility plant modelling}

The utilities consist of a power and heat generation block, which includes one or several gas turbines combined with a waste heat recovery process, and a cooling system, which is a heat exchanger network where seawater is pumped on-site (Table B.8). The component efficiencies, air-to-fuel and pressure ratios 
425 were adapted to reproduce the characteristics of the LM2500+ model of General Electric [26]. This aero426 derivative gas turbine is common in offshore applications: it is characterised by an ISO base rating of 31.9 MW, a gross efficiency of $38.8 \%$ (LHV) and an exhaust temperature of $525^{\circ} \mathrm{C}$. The heat recovery system consists of an indirect heating loop working with hot water, while the cooling system is a direct cooling loop with seawater.

Table B.8: Design parameters of the utility system

\begin{tabular}{lll}
\hline Process & Parameter & Value \\
\hline Cooling system & Working fluid & Seawater \\
& Inlet temperature & $25^{\circ} \mathrm{C}$ \\
& Discharge temperature & $45^{\circ} \mathrm{C}$ \\
Gas turbine & Pressure ratio & $23: 1$ \\
& Compressor efficiency & $85 \%$ \\
& Turbine efficiency & $90 \%$ \\
& Air-to-fuel ratio & 45 \\
Waste heat recovery & Exit temperature & $120^{\circ} \mathrm{C}$ \\
\hline
\end{tabular}

